\title{
El uso de otolitos y huesos de la cabeza para la identificación de dos especies del género Merluccius, en estudios de predador-presa
}

The use of otoliths and bones of the head for the identification of two species of the genus Merluccius, in studies of prey-predator

\author{
Sergio Matías Delpiani ${ }^{1,2}$, Mariano González-Castro ${ }^{1,2}$ y \\ Juan Martín Díaz de Astarloa ${ }^{1,2}$
}

\begin{abstract}
${ }^{1}$ Grupo de Biotaxonomía Morfológica y Molecular de Peces, IIMyC- CONICET, Facultad de Ciencias Exactas y Naturales, Universidad Nacional de Mar del Plata, Funes 3350, B7602AYL Mar del Plata, República Argentina

${ }^{2}$ Consejo Nacional de Investigaciones Científicas y Técnicas (CONICET), Rivadavia 1917, Buenos Aires, República Argentina sdelpiani@mdp.edu.ar
\end{abstract}

\begin{abstract}
A comparative osteological analysis of 6 bones and the sagitta otolith in Merluccius hubbsi and M. australis, two species that live in Southwest Atlantic, has been carried out. The objective was to contribute to identifying these two species as prey in the diet studies, and further description of these bony elements for a better taxonomic identification. Fresh bones disarticulation techniques were used. Potentially diagnostic features were observed for discrimination of species in the following elements: urohyal, maxilla, premaxilla, dentary, quadrate, hyomandibular, and the sagitta otolith.
\end{abstract}

Key words: Hake, osteology, piscivory, prey

\section{INTRODUCCIÓN}

El género Merluccius constituye uno de los grupos de peces demersales más intensamente explotados y al mismo tiempo consumido por un gran número de especies piscívoras como la raya hocicuda, Zearaja chilensis, el pez ángel, Squatina guggenheim, el bacalao criollo, Salilota australis, el abadejo Genypterus blacodes, (Cousseau \& Perrotta 2004), la gaviota austral, Larus scoresbii (Gandini et al. 1999), el pingüino de Magallanes, Spheniscus magellanicus (Frere et al. 1996), la tonina overa, Cephalorhynchus commersonii, el delfín obscuro, Lagenorhynchus obscurus, y el lobo marino de un pelo, Otaria flavescens (Bastida \& Rodríguez 2003), entre otras especies. Este género es considerado problemático en lo que a diferenciación taxonómica se refiere (Inada 1990), más aún cuando se trata de discriminación de especies en contenidos estomacales. En aguas argentinas Merluccius está representado por dos especies, $M$. hubbsi Marini, 1933 y M. australis (Hutton, 1872) (Cousseau \& Perrotta 2004). Sin embargo, una tercera especie (M. patagonicus) fue descripta por Lloris \& Matallanas (2003), la cual es considerada como un sinónimo júnior de M. hubbsi (Díaz de Astarloa et al. 2011, Eschmeyer \& Fricke 2012).
Los caracteres osteológicos han sido utilizados ampliamente en los peces como fundamento para distinguir especies (Chang et al. 1970, Lloris \& Matallanas 2003, Díaz de Astarloa 2005, Dyer 2006, Deli Antoni et al. 2008). Muchos estudios dietarios de animales piscívoros requieren del análisis de restos duros, hallados en heces o en los estómagos (escamas, huesos, otolitos, etc.), para la identificación de las presas (Recchia \& Read 1989, Pierce \& Boyle 1991, Favero et al. 2001), al igual que en los estudios de arqueología de asentamientos litorales de cazadores recolectores, abordando evidencias de fauna marina capturada (Torres 2009, Tivoli \& Zangrando 2011). Ya es reconocido que los otolitos se encuentran entre las estructuras más utilizadas para la identificación de los peces presas (Pierce \& Boyle 1991, Tombari et al. 2000, Volpedo \& Echeverría 2003). Además, los huesos que participan en la captura de la presa, los de la cabeza (premaxilar, maxilar, dentario, etc.) al igual que las vértebras presentan una gran mineralización (fosfato de calcio), esta característica los hace más resistentes a la digestión que otros huesos, de esta manera permanecen un largo tiempo en el estómago y al mismo tiempo son fáciles de identificar (Butler \& Schroeder, 1998). No obstante, son escasos los trabajos que hasta la fecha han empleado la osteología 
como herramienta para la diferenciación de especies del género Merluccius (Inada 1981, Lloris \& Matallanas 2003, Diaz de Astarloa et al. 2011). El objetivo de este trabajo fue contribuir a la identificación de Merluccius hubbsi y M. australis como presas en los estudios de dieta y/o arqueológicos, a través de la profundización en la descripción de elementos óseos de las mismas para su mejor identificación taxonómica.

\section{MATERIALES Y MÉTODOS}

A partir de la captura de 209 especímenes de Merluccius hubbsi (190-995 mm longitud total (LT)) y 32 especímenes de $M$. australis (542-843 mm LT), para su análisis osteológico fueron seleccionados 64 ejemplares de $M$. hubbsi y 3 ejemplares de $M$. australis. Los especímenes fueron colectados en el golfo San Jorge, entre $43^{\circ}$ y $48^{\circ} \mathrm{S}$ (M. hubbsi) y en el área sur patagónica entre $48^{\circ}$ y $55^{\circ} \mathrm{S}$ (M. australis) durante el período febrero 2004-febrero 2005.

Para el análisis osteológico se utilizó la técnica de desarticulación propuesta por Díaz de Astarloa (2005). Se realizó un examen previo en todos los elementos craneales y se seleccionaron 6 elementos óseos y el otolito sagitta, basándose en diferencias osteológicas más destacadas del esqueleto craneal de dos especies nominales presentes en aguas del Atlántico Sudoccidental (M. australis y M. hubbsi).

Se realizó una descripción para cada elemento óseo, sobre la base de las características comunes a las dos especies (M. australis y M. hubbsi) y se describieron las características diferenciales entre éstas dos especies nominales. Para la nomenclatura de los mismos se siguió a Rojo (1976). Las observaciones de los elementos óseos se realizaron a simple vista y bajo lupa estereoscópica Kyowa ${ }^{\circledR}$ SZM, según el tamaño del elemento analizado.

\section{RESULTADOS Y DISCUSIÓN}

Las diferencias osteológicas más destacadas del esqueleto craneal de dos especies nominales presentes en aguas del Atlántico Sudoccidental (M. australis y $M$. hubbsi), estuvieron dadas en los siguientes huesos: urohial, maxilar, premaxilar, dentario, cuadrado, hiomandibular y el otolito sagitta.

\section{DESCRIPCIÓN OSTEOLÓGICA}

El premaxilar constituye el borde superior de la boca. Presenta una región proximal constituida por una sínfisis premaxilar, con dos apófisis; por detrás se encuentra la región distal en forma de media luna que se incurva hacia abajo. Anteriormente, se observa la apófisis premaxilar diferenciándose una parte superior y una inferior con sus extremos redondeados, posteriormente se encuentra la apófisis maxilar. A dos tercios de la longitud se observa una apófisis alar puntiaguda dirigida hacia atrás y que termina posteriormente en forma aguzada. En el borde inferior del hueso se observan dos hileras de alvéolos dentarios y sus dientes. (Fig. 1A, D). En M. hubbsi la apófisis maxilar es ovalada (Fig. 1B) y en M. australis es rectangular (Fig. 1C). En la base de dicha apófisis se observa una pequeña concavidad en M. hubbsi (Fig. 1E) y M. australis un foramen bien desarrollado (Fig. 1F).

El maxilar es un hueso edéntulo, presenta una región proximal y una distal, ligeramente curvadas hacia adentro. La región proximal presenta una cavidad donde articula con la apófisis maxilar del premaxilar (Fig. 1G, J). En M. hubbsi la cavidad donde articula con el premaxilar es triangular (Fig. 1H), siendo rectangular en M. australis (Fig. 1I). La parte inferior del hueso carece de foramen en M. hubbsi (Fig. 1K) y en M. australis presenta un foramen bien notorio dirigido hacia delante (Fig. 1L).

El dentario forma parte de la mandíbula inferior, lleva 2 hileras de dientes largos, curvados hacia adentro y atrás. En la cara externa, en el tercio posterior se evidencia una depresión triangular con un orificio prominente. Su cara interna es lisa y cóncava, presenta una depresión semilunar en la mitad posterior y próxima a la sínfisis dentaria, se extiende una región triangular pequeña con un foramen mentoneano (Fig. 2A). En M. hubbsi se observa una simple depresión semilunar (Fig. 2B), en tanto que en $M$. australis, se distingue una depresión que se extiende hacia la parte anterior del hueso (Fig. 2C).

El urohial es un hueso en forma de $\mathrm{L}$ y posee una base plana. Los extremos de la base son ahusados. Anteriormente posee una proceso anterodorsal (Fig. 2D). Este hueso se caracteriza por presentar una gran variabilidad de osificación en relación a la longitud. En $M$. hubbsi se observa un proceso anterodorsal de mayor envergadura y una fuerte osificación del hueso (Fig. 2E). En tanto que, en $M$. australis es pobremente osificado y de menor tamaño en relación al hueso (Fig. 2F). El grado de osificación en el urohial se ha utilizado para la discriminación de especies del género Merluccius (Inada 1990). Inada (1981, 1990) menciona para M. hubbsi un urohial fuertemente osificado y de forma triangular en sección transversal, en ejemplares mayores a 500 mm LT, pero delgado (poca osificación) y de forma de T invertida en ejemplares de $\sim 250 \mathrm{~mm}$ LT. Por su parte el urohial de 


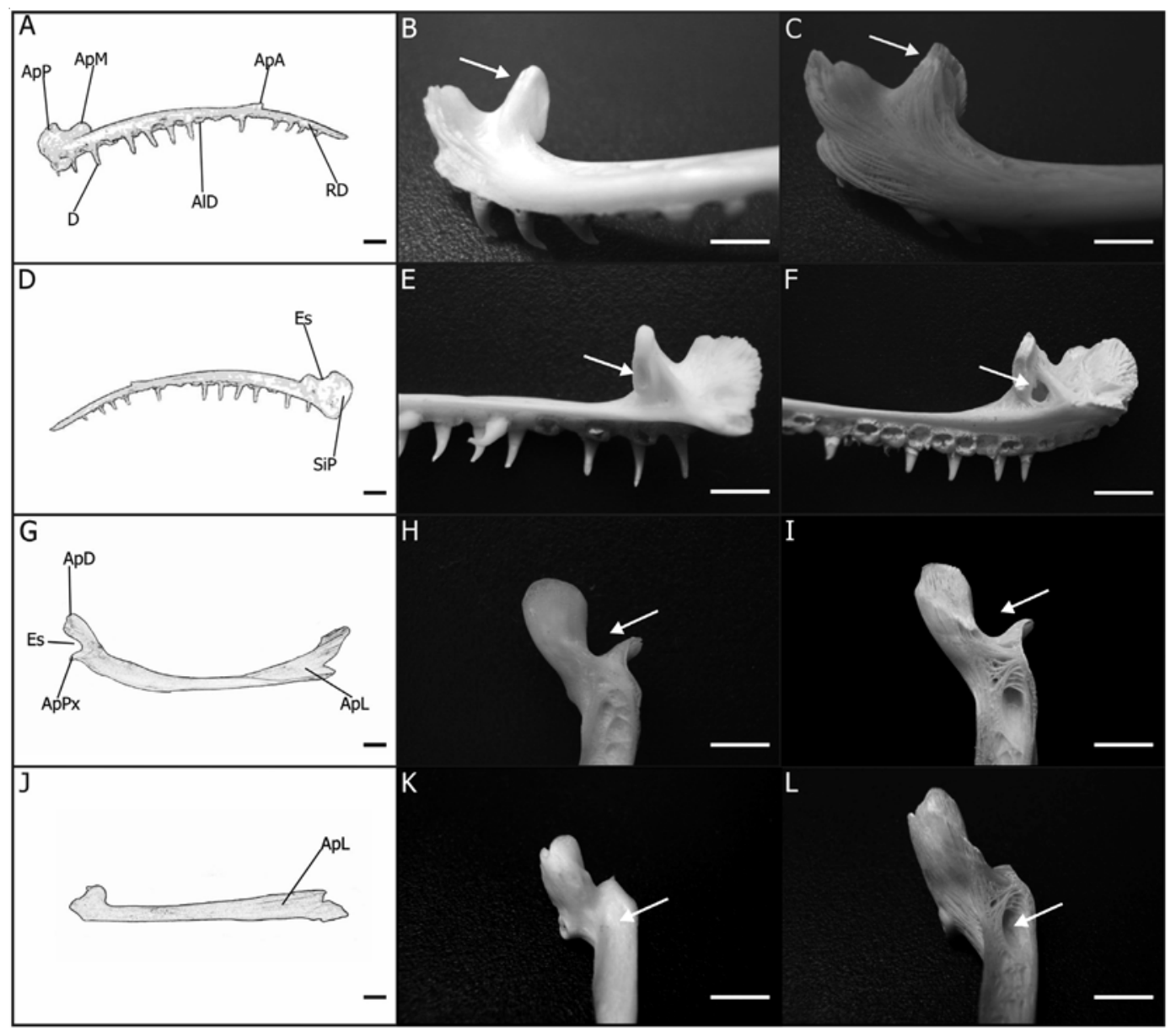

Figura 1. A-F. Premaxilar de Merluccius hubbsi y M. australis; (A). Esquema del premaxilar cara externa AID: alvéolo dentario, ApA: apófisis alar, ApM: apófisis maxilar, ApP: apófisis premaxilar, RD: región distal, D: diente; cara externa del premaxilar de $M$. hubbsi (B) y $M$. australis (C) con diferentes morfologías en su apófisis maxilar (flecha). (D). Esquema del premaxilar cara interna, Es: escotadura, SiP: sínfisis premaxilar; cara interna del premaxilar sin foramen (E) de $M$. hubbsi (flecha) y con foramen (F) de $M$. australis (flecha). G-L. Maxilar de $M$. hubbsi y M. australis; (G). Esquema del hueso maxilar cara dorsal, ApD: apófisis distal; ApL: apófisis laminar, ApPx: apófisis proximal, Es: escotadura; detalle del maxilar con escotadura triangular (H) de $M$. hubssi (flecha) y rectangular (I) M. australis (flecha); ( $\mathrm{J}$. hueso maxilar cara externa, ApL: apófisis laminar; detalle del maxilar sin foramen (K) de $M$. hubbsi (flecha) y con foramen (L) $M$. australis (flecha). Barra de escala $\mathbf{5} \mathbf{5 m} /$ A-F. Premaxilla of Merluccius hubbsi and M. australis; (A). Schematic of premaxilla outer face AID: dental alveolus, ApA: apophyses wings, ApM maxillary process, ApP: premaxilla apophyses, RD: distant region, D: tooth; Outer surface of the premaxilla of $M$. hubbsi (B) and $M$. australis (C) with different morphological maxillary processes (arrow); (D). Schematic of premaxilla inner face, Es: notch, SiP: premaxilar symphysis; inner surface of the premaxilla without foramen (E) in M. hubbsi (arrow) and with foramen (F) in M. australis (arrow). G-L. Maxilla of M. hubbsi and $M$. australis; (G). Schematic of maxilla dorsal face, ApD: distal apophysis, ApL: laminar apophysis, ApPx: proximal apophysis, Es: notch; detail of the maxilla with a triangular notch $(H)$ in $M$. hubbsi (arrow) and rectangular (I) in M. australis (arrow); $(\mathrm{J})$. Schematic of maxilla external face, ApL: laminar apophysis; detail of the maxilla without foramen $(\mathrm{K})$ in $M$. hubbsi (arrow) and with foramen (L) in M. australis (arrow) Scale bar $=5 \mathrm{~mm}$ 


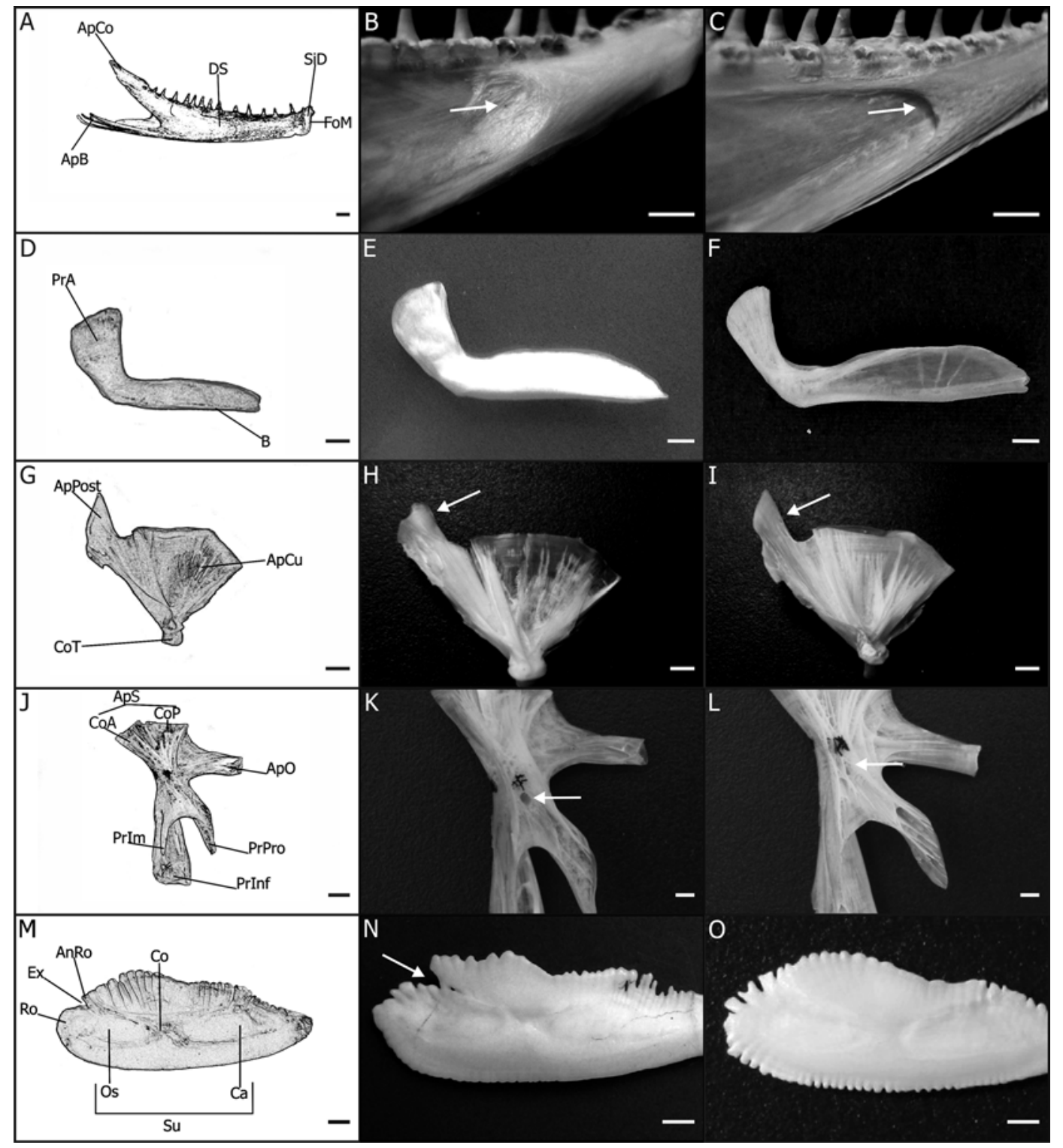

Figura 2. A-C. Dentario de Merluccius hubbsi y M. australis; (A). Esquema del dentario cara interna, ApB: apófisis basal, ApCo: apófisis coronoide, DS: depresión semilunar, FoM: foramen mentoneano, SiD: sínfisis dentaria; dentario con leve depresión semilunar (B) en $M$. hubbsi y con cavidad semilunar (C) en $M$. australis. D-F. Urohial de $M$. hubbsi y $M$. australis; (D). Detalle del urohial cara externa B: base y PrA: proceso anterodorsal; en $M$. hubbsi (E) se observa un urohial fuertemente calcificado y un proceso anterodeorsal de mayor envergadura que en $M$. australis (F). G-I. Cuadrado de M. hubbsi y M. australis; (G). Esquema del cuadrado cara interna ApCu: apófisis cuadrangular, ApPost: apófisis posteroventral, CoT: cóndilo transversal; cuadrado con apófisis posteroventral redondeada (H) en $M$. hubbsi y ahusada (I) de M. australis. JL. Hiomandibular de M. hubbsi y M. australis; (J). Esquema del hiomandibular cara externa ApS: apófisis superior, ApO: apófisis opercular, PrInf: proceso inferior, CoA: cóndilo anterior, CoP: cóndilo posterior, PrIm: proceso intermuscular, PrPro: proceso preopercular; hiomandibular con foramen (K) en M. hubbsi (flecha) y sin foramen (L) de M. australis. M-O. Sagitta de M. hubbsi y M. australis. (M). Características del sagitta derecha (cara interna) de M. hubbsi. AnRo: anti-rostrum, Ca: cauda, Co: collum, Ex: excisura, Os: ostium, Ro: rostrum, Su: sulcus; Sagitta de $M$. hubbsi (N) de $750 \mathrm{~mm}$ LT y Sagitta de M. australis (O) de $822 \mathrm{~mm}$ LT. Barra de escala = $5 \mathrm{~mm}$ / A-C. Dentary of Merluccius hubbsi and M. australis; (A). Diagram of dentary, inner face ApB: basal apophysis, ApCo: coronoid apophysis, DS: semilunar depression, FoM: mentonean foramen, SiD: dentary symphysis; dentary with smooth semilunar depression in (B) M. hubbsi and semilunar cavity (C) M. australis. D-F. Urohyal of M. hubbsi and M. australis; (D). Schematic of urohyal bone outer face, B: base and PrA: anterodorsal process; (E) in M. hubbsi shows a strongly calcified urohyal and anterodorsal process larger than (F) M. australis; G-I. Quadrate of M. hubbsi and M. australis. (G). Schematic of quadrate bone outer face, ApCu: quadrangular apophysis, ApPost: posteroventral apophysis, CoT: transverse condyle; quadrate with rounded preopercular apophysis in (H) M. hubbsi and taper-shaped in (I) M. australis. J-L. Hyomandibula of M. hubbsi and M. australis; (J). Schematic of hyomandibular bone outer face, ApS superior apophysis, ApO: opercular apophyses, PrInf: inferior process, CoA: anterior condyle, CoP: posterior condyle, PrIm: intermuscular process, PrPro: preopercular process; hyomandibular foramen (K) of M. hubbsi and without foramen in (L) M. australis; M-O. Sagitta of M. hubbsi and M. australis. (M). Schematic of right sagitta otolith (inner face) of M. hubbsi. AnRo: antrirostrum, Ca: cauda, Co: collum, Ex: excisure, Os: ostium, Ro: rostrum, Su: sulcus; Sagitta of M. hubbsi specimen (N) of 750 mm LT; Sagitta of M. australis (O) of $822 \mathrm{~mm} \mathrm{LT}$. Scale bar $=5 \mathrm{~mm}$ 
M. australis es delgado independientemente de la longitud del ejemplar (Inada 1990, Lloris \& Matallanas 2003). En el presente trabajo, el urohial de M. hubbsi presentó un mayor grado de osificación que el de $M$. australis para ejemplares mayores a $500 \mathrm{~mm}$.

El cuadrado es de forma triangular, forma parte del suspensorio de la mandíbula inferior, con un cóndilo doble en el vértice inferior, que se articula con la porción articular de la mandíbula a través de una depresión en forma de silla de montar. La parte media se adelgaza hacia el borde superior, presentando líneas concéntricas de crecimiento que constituyen la apófisis cuadrangular (el hueso mismo). Posteriormente, posee una apófisis posteroventral plana en su base y cóncava a partir de su parte media, que articula con el hueso preopercular. Este último proceso se observa acanalado a partir de su parte media. (Fig. 2G). En M. hubbsi la apófisis posteroventral suele poseer la cara interna de forma redondeada y se encuentra separada por una hendidura que se origina a partir de la parte media a la superficie dorsal de la apófisis cuadrangular. (Fig. $2 \mathrm{H})$. En M. australis el borde interno de la apófisis posteroventral es laminar, de forma ahusada y con un borde ondulado. Las apófisis cuadrangular y posteroventral se encuentran fuertemente unidas (Fig. 2I). Dichos caracteres presentes en el premaxilar, maxilar, dentario, urohial y cuadrado, no han sido mencionados por otros autores.

El hiomandibular es de forma irregular con 4 apófisis de tamaño y forma variables. Este hueso presenta en su cara externa una apófisis superior o auricular caracterizada por ser ancha y grande. Posterior a ésta se proyecta la apófisis opercular, de forma cilíndrica. En el mismo plano que las apófisis descritas previamente, se encuentra el proceso inferior, que se opone a la apófisis superior formando la región inferior del hueso, la cual es aplanada, triangular. En el punto de origen de la apófisis opercular surge una estructura que se bifurca en dos procesos: uno anterior llamado proceso intermuscular, que es delgado y termina en forma ahusada, y uno posterior denominado proceso preopercular, que es aplanado en su cara lateral (Fig. 2J). En M. hubbsi el proceso preopercular es aproximadamente perpendicular al eje vertical del hueso y no presenta foramen en la intercepción de los procesos intermuscular y preopercular (Fig. 2K), en tanto que M. australis presenta el proceso intermuscular con una leve inclinación hacia abajo. Esta última presenta un foramen bien desarrollado en el origen de los procesos intermuscular y preopercular (Fig. 2L). La longitud del proceso intermuscular del hiomandibular se utilizó en el pasado para discriminar entre grupos de especies de Merluccius (Inada 1981). Sin embargo, en este trabajo se encontró que dicho carácter es muy variable dentro de cada una de las especies analizadas, coincidentemente con lo hallado por Díaz de Astarloa et al. (2011).

En el otolito sagitta se pueden diferenciar: rostro, antirostro, excisura, collum, ostium, cauda y sulcus (Fig. 2M). La sagitta presenta forma oblonga con una parte anterior redondeada y una posterior ahusada. En el extremo anterior posee una excisura en forma de $\mathrm{V}$ que coincide con el borde anterior de la cresta dorsal del sulcus. La excisura separa el rostro alto y cóncavo, de un antirostro bajo y agudo. Posee un heterosulcus compuesto por un ostium y una cauda (Fig. 2M). La diferencia más notable entre las especies nominales fue la presencia de un rostro y un anti-rostro separados por una prominente exisura en M. hubbsi (Fig. 2N). En tanto que M. australis presenta un borde anterodorsal dentado y carece de excisura (Fig. 2O). La discriminación de las especies del género Merluccius, sobre la base de la morfología comparativa de los otolitos sagitta, se dificulta en individuos juveniles, pero en ejemplares adultos (que superan la longitud de primera madurez) las diferencias morfológicas son más evidentes. Se encontró que los otolitos sagitta de $M$. australis son más delgados y posteriormente más prominentes en relación a los de $M$. hubbsi, resultados similares fueron observados para las especies $M$. capensis y $M$. paradoxus, M. albidus y M. bilinearis (Botha 1971, Mombeck 1971). Los otolitos sagitta en ejemplares adultos de $M$. hubbsi presentan excisura (Rojo 1976, Inada 1981, Diaz de Astarloa et al. 2011). En el presente trabajo todos los individuos de $M$. hubbsi de longitudes mayores a $600 \mathrm{~mm}$ presentaron excisura, en cambio los ejemplares de $M$. australis carecieron de la misma. El mismo resultado fue observado por Diaz de Astarloa et al. (2011).

La información aportada facilita la identificación de las especies del genero Merluccius en las dietas de las especies piscívoras y sitios arqueológicos en el Atlántico sudoccidental, y demuestra que las diferencias morfológicas de los componentes óseos del cráneo poseen validez diagnóstica. Estos caracteres serán un importante complemento a los morfométricos y merísticos tradicionalmente utilizados. A continuación se resumen los caracteres osteológicos cefálicos distintivos que permiten la separación de las especies de Merluccius analizadas (Tabla 1). 
Tabla 1. Caracteres osteológicos diagnósticos del esqueleto craneal de dos especies del género Merluccius / Osteological diagnostic features of the cranial skeleton of two species of Merluccius

\begin{tabular}{|c|c|c|}
\hline Hueso & M. hubbsi & M. australis \\
\hline Premaxilar & $\begin{array}{l}\text { La apófisis maxilar es más ovalada. Foramen poco } \\
\text { desarrollado o ausente en la base de apófisis } \\
\text { maxilar }\end{array}$ & $\begin{array}{l}\text { La apófisis maxilar es rectangular. Foramen bien } \\
\text { desarrollado en la base de apófisis maxilar }\end{array}$ \\
\hline Maxilar & $\begin{array}{l}\text { Cavidad donde articula con el premaxilar es de } \\
\text { forma triangular. Foramen anteroventral poco } \\
\text { desarrollado o ausente }\end{array}$ & $\begin{array}{l}\text { Cavidad donde articula con el premaxilar es } \\
\text { rectangular. Foramen anteroventral bien desarrollado } \\
\text { dirigido hacia delante }\end{array}$ \\
\hline Dentario & $\begin{array}{l}\text { Depresión semilunar, se observa como una pequeña } \\
\text { depresión }\end{array}$ & $\begin{array}{l}\text { Depresión semilunar continua hacia delante en el } \\
\text { hueso }\end{array}$ \\
\hline Urohial & $\begin{array}{l}\text { Proceso anterodorsal de mayor envergadura. Fuerte } \\
\text { osificación del hueso }\end{array}$ & $\begin{array}{l}\text { Proceso anterodorsal de menor envergadura. Escasa } \\
\text { osificación del hueso }\end{array}$ \\
\hline Cuadrado & $\begin{array}{l}\text { La apófisis posteroventral, suele poseer la cara } \\
\text { interna de forma redondeada; ésta se encuentra } \\
\text { separada por una hendidura que se origina a partir } \\
\text { de la parte media a la superficie dorsal de la } \\
\text { apófisis cuadrangular }\end{array}$ & $\begin{array}{l}\text { El borde interno de la apófisis posteroventral es } \\
\text { laminar de forma ahusada y con un borde ondulado. } \\
\mathrm{La} \text { apófisis cuadrangular y posteroventral se } \\
\text { encuentran fuertemente unidas }\end{array}$ \\
\hline Hiomandibular & $\begin{array}{l}\text { Proceso preopercular es perpendicular al eje } \\
\text { vertical del hueso. Presentan un foramen poco } \\
\text { desarrollado o ausente }\end{array}$ & $\begin{array}{l}\text { Proceso preopercular tiene una leve inclinación hacia } \\
\text { abajo. Presentan un foramen bien desarrollado en la } \\
\text { parte inferior }\end{array}$ \\
\hline Otolito (sagitta) & Presencia de excisura ostii & No presenta excisura ostii \\
\hline
\end{tabular}

\section{Agradecimientos}

Agradecemos a los colegas y tripulantes de los BIP 'Dr. Eduardo L. Holmberg' y 'Cap. Oca Balda’ del Instituto Nacional de Investigación y Desarrollo Pesquero (INIDEP) por la desinteresada colaboración en la colecta de los peces.

\section{LITERATURA CITADA}

Bastida R \& D Rodríguez. 2003. Mamíferos marinos de Patagonia y Antártida, 208 pp. Vazquez Mazzini Editores, Buenos Aires.

Botha L. 1971. Growth and otolith morphology of the Cape hakes Merluccius capensis Cast. and M. paradoxus Franca. Investigational Report Division of Sea Fisheries of South Africa 97: 1-32.

Butler V \& R Schroeder. 1998. Do digestive processes leave diagnostic traces on fish bones? Journal of Archaeological Science 25(10): 957-971.

Chang A, G Arratia \& V Alegría. 1970. Osteología de Merluccius gayi (Guichenot, 1848). Boletín del Museo Nacional de Historia Natural, Chile 32: 299-374.

Cousseau MB \& RG Perrotta. 2004. Peces marinos de Argentina. Biología, distribución, pesca, 167 pp. Publicaciones Especiales INIDEP, Mar del Plata.
Deli Antoni MY, M González-Castro \& JM Díaz de Astarloa. 2008. Análisis de la morfología ósea de peces de la laguna costera Mar Chiquita, Argentina. Revista de Biología Marina y Oceanografía 43(2): 355-380.

Díaz de Astarloa JM. 2005. Osteología craneal comparada de tres especies de lenguado del género Paralichthys (Pleuronectiformes, Paralichthyiidae) del Atlántico suroccidental. Revista Chilena de Historia Natural 78(3): 343-391.

Díaz de Astarloa JM, SI Bezzi, M González-Castro, E Mabragaña, D Hernández, SM Delpiani, DE Figueroa, MB Cousseau, MY Deli Antoni \& L Tringali. 2011. Morphological, morphometric, meristic and osteological evidence for two species of hake (Actinopterygii: Gadiformes: Merluccius) in Argentinean waters. Journal of Fish Biology 78: 1336-1358.

Dyer B. 2006. Systematic revision of the South American silversides (Teleostei, Atheriniformes). Biocell 30: 69-88.

Eschmeyer WN \& R Fricke. 2012. Catalog of fishes electronic version, California Academy of Sciences, San Francisco. [on line] <http://research.calacademy.org/ichthyology/ catalog/fishcatmain.asp>

Favero M, S Bachmann, S Copello, RM Jelicich, MP Silva, M Ghys, C Khatchikian \& L Mauco. 2001. Aves marinas del sudeste bonaerense. En: Iribarne O (ed). Reserva Mar Chiquita: características físicas, biológicas y ecológicas, pp 251-267. Editorial Martin, Mar del Plata. 
Frere E, P Gandini \& V Lichtschein. 1996. Variación latitudinal en la dieta del Pingüino de Magallanes (Spheniscus magellanicus) en la costa Patagónica, Argentina. Ornitología Neotropical 7: 35-41.

Gandini PA, JP Seco Pon \& E Frere. 2008. Composición de la dieta de la gaviota austral (Larus scoresbii) en patagonia, Argentina. Ornitologia Neotropical 19: 109-116.

Inada T. 1981. Studies on the Merlucciid Fishes. Bulletin of the Far Seas Fisheries Research Laboratory 18: 1-172.

Inada T. 1990. Merluccidae. En: Cohen DM, T Inada, T Iwamoto \& N Scialabba (eds). FAO Species catalogue. Vol. 10. Gadiform fishes of the world (Order Gadiformes). An annotated and illustrated catalogue of cods, hakes, grenadiers and other gadiform fishes known to date. FAO Fisheries Synopsis 125(10): 327-344.

Lloris D \& J Matallanas. 2003. Description of a new species of hake: Merluccius patagonicus sp. nov. (Gadiformes: Merlucciidae) from the waters of Argentina. Scientia Marina 67: 323-326.

Mombeck F. 1971. Notes on the distinction of Northwest Atlantic hakes, Merluccius albidus and M. bilinearis. ICNAF Research bulletin 8: 87-89.

Pierce GJ \& PR Boyle. 1991. A review of methods for diet analysis in piscivorous marine mammals. Oceanography and Marine Biology: An Annual Review 29: 409-486.
Recchia CA \& AJ Read. 1989. Stomach contents of harbour porpoises, Phocoena phocoena (L.), from the Bay of Fundy. Canadian Journal of Zoology 67: 2140-2146.

Rojo A. 1976. Osteología de la merluza argentina (Merluccius hubbsi, Marini 1933). Boletín del Instituto Español de Oceanografía 219: 1-61.

Tombari AD, AV Volpedo \& DD Echeverría. 2000. Patrones morfológicos de la sagitta de pejerreyes de la ictiofauna argentina. Thalassas 16: 11-19.

Torres J. 2009. La pesca entre los cazadores recolectores terrestres de la isla grande de Tierra del Fuego, desde la prehistoria a tiempos etnográficos. Magallania, Chile 37(2): 109-138.

Tivoli AM \& F Zangrando. 2011. Subsistence variations and landscape use among maritime hunter-gatherers. zooarchaeological analysis from the Beagle Channel (Tierra del Fuego, Argentina). Journal of Archaeological Science 38: $1148-1156$.

Volpedo AV \& DD Echeverria. 2003. Ecomorphological patterns of the sagitta in fish on the continental shelf off Argentine. Fisheries Research 60: 551-560.

Recibido el 1 de diciembre de 2011 y aceptado el 1 de junio de 2012

Editor Asociado: Mauricio Landaeta D. 